\title{
Numerical simulation of thin paint film flow
}

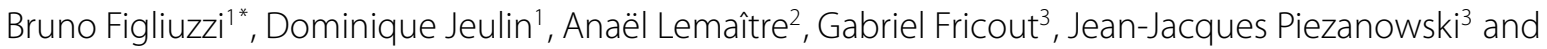 \\ Paul Manneville ${ }^{4}$
}

"Correspondence:
figliuzzi.bruno@gmail.com
${ }^{1}$ Centre de Morphologie
Mathématique, École des Mines
ParisTech, 77300, Fontainebleau,
France
Full list of author information is
available at the end of the article

\begin{abstract}
Purpose: Being able to predict the visual appearance of a painted steel sheet, given its topography before paint application, is of crucial importance for car makers. Accurate modeling of the industrial painting process is required.

Results: The equations describing the leveling of the paint film are complex and their numerical simulation requires advanced mathematical tools, which are described in detail in this paper. Simulations are validated using a large experimental data base obtained with a wavefront sensor developed by Phasics ${ }^{\mathrm{TM}}$.

Conclusions: The conducted simulations are complex and require the development of advanced numerical tools, like those presented in this paper.
\end{abstract}

Keywords: thin films; numerical simulation; industrial painting process; roughness; lubrication approximation.

\section{Introduction}

The visual appearance of painted steel sheets forming the body of a car is a prominent factor in appreciating its quality. Being able to predict it is thus of crucial importance to car makers, while remaining a serious mathematical challenge requiring accurate modeling of the industrial painting process.

The deposition of the successive coating layers on a car body involves complex physical and chemical processes, with many variants. Here, we consider the sheet in its initial surface state (galvanized and phosphated) and summarize the painting process as follows: once assembled, the car body is immersed in an electrophoresis bath, where a layer of corrosion-protecting paint is deposited. The vehicle body is then baked in an oven. A second paint layer, the sealer, is applied and the vehicle baked again. Finally, a layer of lacquer is applied before a last baking. The steel sheet is thus covered with three layers of coating as shown in Figure 1.

The last two paint coatings are mainly designed to provide an aesthetically pleasing appearance to the car. During the painting process, the final topography of each layer results from two main processes:

- the leveling of the film (flow and evaporation) which occurs during the flash time, i.e. the time period just following the end of the deposit,

- baking in an oven, which favors evaporation.

The leveling process has received considerable attention in the literature, although not in the context of the industrial paints used in the automotive industry. In 1961, Orchard [1] was the first to note that the leveling dynamics is controlled by an interplay between 


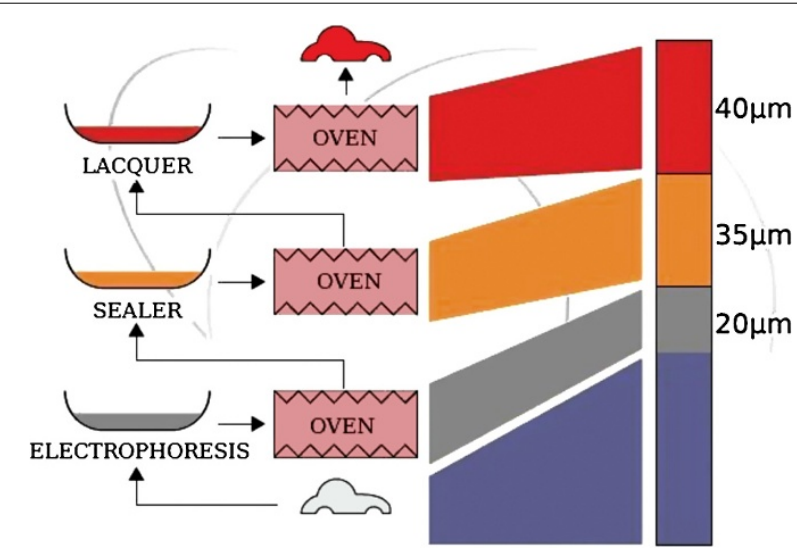

Figure 1 Successive paint layers on a steel sheet, with approximate thicknesses as indicated.

surface tension, with capillary forces tending to reduce surface irregularities, and the fluid viscosity limiting the flow induced by that leveling. Orchard's model is mainly based on two assumptions: the paint exhibits a Newtonian behavior and evaporation effects are negligible. To take into account the effects of evaporation, Overdiep [2] considered a fluid made of a resin and a solvent, where only the solvent can evaporate, demonstrating the potential importance of the surface tension spatial variations. Surface tension indeed depends on the paint composition, in particular on the respective proportions of resin and solvent. In the presence of evaporation, thinner regions tend to dry faster, and therefore to have lower solvent concentrations, which causes surface tension gradients, a physical phenomenon known as Marangoni effect, hence a shearing effect at the film surface, understood as the main physical effect involved in the leveling of the paint film by Overdiep. This approach was taken up and developed in several subsequent articles. Wilson [3] and later Howison et al. [4] analyzed and generalized Overdiep's model, performing numerical simulations that showed good agreement with experimental data collected for simple deposit geometries.

The topography of the substrate on which the coating is deposited plays an important role in the flow dynamics. In 1995, Weidner et al. [5] studied the effect of substrate curvature on the film flow in a two-dimensional context. Subsequently Eres et al. [6] and later Schwartz et al. [7] generalized the work to the three-dimensional case. In these papers, numerical models have been implemented for specific topographies, showing good agreement with experimental measurements. Gaskell et al. [8, 9] finally considered the generalization of the different models to the case of inclined substrates, where gravity plays a significant physical role in the flow dynamics.

Industrial paints used in the context of the automotive industry are complex media that have not been extensively studied. Their detailed rheology is not well known, though its effects on the leveling are a key issue. In view of the complexity of the phenomena, experiments aiming at the identification of the physical effects within the film and the evaluation of their relative importance appear to be a prerequisite to film flow modeling. Using a wavefront sensor developed by Phasics ${ }^{\mathrm{mat}}[10]$, we could determine the evolution of rough surfaces accurately and with a high temporal resolution throughout the whole painting process [11]. In Section 2, we describe the mathematical model used to model the evolution of the painted film topography and its numerical simulation. Section 3 is devoted to 


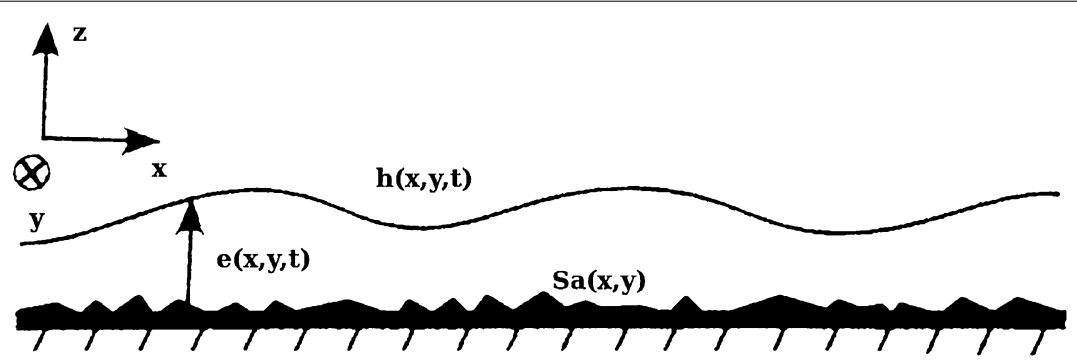

Figure 2 Thin paint film flow.

the presentation of the experimental data obtained with the wavefront sensor. Rheological parameters extracted from the experimental data are used in Section 4 to perform a simulation of the topography evolution during the painting process. Conclusions are drawn in Section 5.

\section{The mathematical model and its implementation}

Following the accepted practice, we study the leveling process within the framework of a lubrication approximation, but more elaborate theories can be developed from the NavierStokes equations [12-17]. The lubrication approximation builds on two observations: firstly, the thin film flow is very slow, so that it becomes possible to neglect the inertia terms in the Navier-Stokes equation; secondly, the thickness of the film is much smaller than the wavelength of the modulations along the surface, which also implies that the fluid velocity is essentially directed parallel the surface. All this allows a substantial simplification of the equations describing the flow of the thin paint film.

\subsection{Physical model}

Here, we consider the leveling of a thin incompressible film deposited on an horizontal steel sheet, as represented on Figure 2. The topography of the bare sheet is denoted as $S_{\mathrm{a}}(x, y)$, the film thickness as $e(x, y, t)$, and the height of film free surface as $h(x, y, t)$. The paint film is deposited at $t=0$, and evolves until solidification due to polymer curing, which happens at $t_{\text {ret }}$ during the baking. The final film height is then $S_{\mathrm{a}}(x)+e\left(x, t_{\mathrm{ret}}\right)$. The film thickness at the beginning of the leveling is approximately $H=70 \mu \mathrm{m}$. A typical value of the paint velocity is $U=10 \mu \mathrm{m} / \mathrm{s}$. The Reynolds number $R e=\rho U H / \eta$ is approximately $R e \cong 7.8 \times 10^{-7} \ll 1$. It is also of interest to compute the Ohnesorge number of the film flow, which relates the viscous forces to inertial and surface tension forces:

$$
O h=\frac{\eta}{\rho \gamma L}
$$

where $L$ denotes a characteristic length in the horizontal direction. With $\eta=0.9$ Pa.s, $\rho=$ $1000 \mathrm{~kg} / \mathrm{m}^{3}, \gamma=2.71 \times 10^{-2} \mathrm{~N} / \mathrm{m}$ and $L=150 \mu \mathrm{m}$, we find $O h \cong 221$, which indicates a preponderant influence of the viscosity in the leveling phenomenon.

\subsubsection{Lubrication approximation}

Without making any assumption about the paint rheology, neglecting gravity, the mechanical equilibrium equation reads

$$
-\nabla p+\nabla \cdot \overline{\bar{\sigma}}=0,
$$


where $\overline{\bar{\sigma}}$ denotes the deviator stress tensor and $p$ the local pressure within the film. Letting $u$ and $v$ be the velocity components along $x$ and $y$, the $z$-component being neglected in the lubrication approximation, the strain rate tensor reads:

$$
\frac{1}{2}\left(\nabla \mathbf{u}+{ }^{T} \nabla \mathbf{u}\right)=\left(\begin{array}{ccc}
\frac{\partial u}{\partial x} & \frac{1}{2}\left(\frac{\partial u}{\partial y}+\frac{\partial v}{\partial x}\right) & \frac{1}{2} \frac{\partial u}{\partial z} \\
\frac{1}{2}\left(\frac{\partial u}{\partial y}+\frac{\partial \nu}{\partial x}\right) & \frac{\partial v}{\partial y} & \frac{1}{2} \frac{\partial v}{\partial z} \\
\frac{1}{2} \frac{\partial u}{\partial z} & \frac{1}{2} \frac{\partial \nu}{\partial z} & 0
\end{array}\right) .
$$

Within the lubrication approximation, the gradients of $u$ and $v$ along $x$ and $y$ can be neglected. The strain rate tensor is then reduced to:

$$
\frac{1}{2}\left(\nabla \mathbf{u}+{ }^{T} \nabla \mathbf{u}\right)=\left(\begin{array}{ccc}
0 & 0 & \frac{1}{2} \frac{\partial u}{\partial z} \\
0 & 0 & \frac{1}{2} \frac{\partial v}{\partial z} \\
\frac{1}{2} \frac{\partial u}{\partial z} & \frac{1}{2} \frac{\partial v}{\partial z} & 0
\end{array}\right)
$$

One can expect the deviator stress tensor to be parallel to the strain rate tensor. Tensor $\overline{\bar{\sigma}}$ then reads

$$
\overline{\bar{\sigma}}=\left(\begin{array}{ccc}
0 & 0 & \sigma_{x z} \\
0 & 0 & \sigma_{y z} \\
\sigma_{x z} & \sigma_{y z} & 0
\end{array}\right)
$$

so that Equation 1 becomes:

$$
\left\{\begin{array}{l}
-\frac{\partial p}{\partial x}+\frac{\partial \sigma_{x z}}{\partial z}=0 \\
-\frac{\partial p}{\partial y}+\frac{\partial \sigma_{y z}}{\partial z}=0 \\
-\frac{\partial p}{\partial z}=0
\end{array}\right.
$$

Boundary conditions are given by a no slip kinematic condition at the substrate surface, $u(z=0)=v(z=0)=0$, and by a mechanical condition expressing that the constraint is zero at the free surface, $\sigma_{x z}(z=h)=\sigma_{y z}(z=h)=0$. In what follows, we will set the origin of the altitudes at the mean substrate level. Equation 5 can consequently be integrated to yield:

$$
\left\{\begin{array}{l}
\sigma_{x z}=-\frac{\partial p}{\partial x}(h-z) \\
\sigma_{y z}=-\frac{\partial p}{\partial y}(h-z) .
\end{array}\right.
$$

The pressure is given as the product of the surface tension and the free surface curvature $C$ which at lowest order reads:

$$
C=-\frac{\partial^{2} h}{\partial x^{2}}-\frac{\partial^{2} h}{\partial y^{2}}
$$

where $h(x, y, t)=e(x, y, t)+S_{\mathrm{a}}(x, y)$ is the altitude of the fluid surface. Finally, the local altitude is linked to the evaporation rate $E$ and the local flow rate $\mathbf{q}$ by the mass conservation 
equation

$$
\frac{\partial h}{\partial t}(x, y, t)=-\nabla_{\mathbf{h}} \cdot \mathbf{q}(x, y, t)-E(x, y, t)
$$

where $\nabla_{\mathrm{h}}$ is the gradient along the plane $(x, y)$.

\subsubsection{Paint rheology}

Equations 6-8 have been derived without making any assumptions about the paint rheology. To close these equations, we have to prescribe how the mass flux $\mathbf{q}$ depends on the local pressure gradient.

In [11], $\mathbf{q}$ was computed from the data obtained with the wavefront sensor by solving the following problem:

$$
-\nabla_{\mathrm{h}}\left(\frac{\partial h}{\partial t}(x, y, t)+E(x, y, t)\right)=\Delta_{\mathrm{h}} \mathbf{q}(x, y, t)
$$

which comes after noting that within the lubrication approximation:

$$
\operatorname{curl} \operatorname{curl}(\mathbf{q})=\mathbf{0}
$$

Estimating the left hand side of Equation 9 indeed allows the access to the local values of the mass flux by solving the Poisson equation, and hence permits us to test the rheological model. The so-obtained data showed that for the space and time scales involved in the problem, the film can be considered as Newtonian.

Assuming a Newtonian rheology, the deviator stress tensor can then easily be expressed as a function of the strain rate tensor:

$$
\overline{\bar{\sigma}}=\frac{\eta}{2}\left(\nabla \mathbf{u}+{ }^{T} \nabla \mathbf{u}\right)
$$

so that Equation 5 can be rewritten as:

$$
\left\{\begin{array}{l}
-\frac{\partial p}{\partial x}(x, y, t)+\eta \frac{\partial^{2} u}{\partial z^{2}}(x, y, t)=0 \\
-\frac{\partial p}{\partial y}(x, y, t)+\eta \frac{\partial^{2} v}{\partial z^{2}}(x, y, t)=0 .
\end{array}\right.
$$

\subsubsection{Newtonian model equation}

Since the pressure $p$ is independent of $z$, Equations 12 can easily be integrated. Boundary conditions were indeed given by a no slip kinematic condition at the substrate surface, $u\left(z=S_{\mathrm{a}}\right)=0, v\left(z=S_{\mathrm{a}}\right)=0$, and by a mechanical condition expressing that the constraint is zero at the free surface, $\partial u / \partial z(z=h)=0, \partial v / \partial z(z=h)=0$.

$$
\left\{\begin{array}{l}
u(x, y, z, t)=\frac{1}{\eta} \frac{\partial p}{\partial x}(x, y, t)\left(\frac{1}{2} z^{2}-h\left(z-S_{\mathrm{a}}\right)-\frac{1}{2} S_{\mathrm{a}}^{2}\right), \\
v(x, y, z, t)=\frac{1}{\eta} \frac{\partial p}{\partial y}(x, y, t)\left(\frac{1}{2} z^{2}-h\left(z-S_{\mathrm{a}}\right)-\frac{1}{2} S_{\mathrm{a}}^{2}\right) .
\end{array}\right.
$$


Consequently, the local flow components on the film thickness along the horizontal directions read

$$
\left\{\begin{array}{l}
q_{x}=\int_{S_{\mathrm{a}}}^{h} u(x, y, z, t) d z=\frac{\gamma}{3 \eta}\left(h-S_{\mathrm{a}}\right)^{3}\left(\frac{\partial^{3} h}{\partial x^{3}}+\frac{\partial^{3} h}{\partial x \partial y^{2}}\right), \\
q_{y}=\int_{S_{\mathrm{a}}}^{h} v(x, y, z, t) d z=\frac{\gamma}{3 \eta}\left(h-S_{\mathrm{a}}\right)^{3}\left(\frac{\partial^{3} h}{\partial y^{3}}+\frac{\partial^{3} h}{\partial y \partial x^{2}}\right) .
\end{array}\right.
$$

Using the mass conservation Equation 8, the complete model equation is

$$
\begin{aligned}
\frac{\partial h}{\partial t}= & -\frac{\gamma}{3 \eta} \frac{\partial}{\partial x}\left[\left(h-S_{\mathrm{a}}\right)^{3}\left(\frac{\partial^{3} h}{\partial x^{3}}+\frac{\partial^{3} h}{\partial x \partial y^{2}}\right)\right] \\
& -\frac{\gamma}{3 \eta} \frac{\partial}{\partial y}\left[\left(h-S_{\mathrm{a}}\right)^{3}\left(\frac{\partial^{3} h}{\partial y^{3}}+\frac{\partial^{3} h}{\partial y \partial x^{2}}\right)\right]-E .
\end{aligned}
$$

We will assume that the paint is composed of a resin in concentration $1-c$ and a solvent in concentration $c$. Only the solvent can evaporate, while the evaporation rate will essentially depend on the solvent concentration. Accordingly, we shall assume that the largest scales patterns attenuation is mainly caused by evaporation, for a leveling caused by surface tension would suppose a huge mass transport which would be unrealistic considering the geometric characteristics of the painted film. A method based on this idea is presented in [11], which allows a determination of the evaporation rate as a function of $c$. If we neglect the local variations of the solvent concentration, the evaporation rate will consequently be spatially constant, and will only vary with time.

\subsubsection{Marangoni effect}

The local variations in the solvent concentration may generate a surface tension gradient. This surface tension gradient modifies the mechanical equilibrium conditions on the free film surface which become

$$
\eta \frac{\partial u}{\partial z}=\frac{\partial \gamma}{\partial x}, \quad \eta \frac{\partial v}{\partial z}=\frac{\partial \gamma}{\partial y} .
$$

Expressions 14 become then

$$
\left\{\begin{array}{l}
q_{x}=\frac{1}{3 \eta}\left(h-S_{\mathrm{a}}\right)^{3} \frac{\partial p}{\partial x}+\frac{1}{2 \eta} \frac{\partial \gamma}{\partial x}\left(h-S_{\mathrm{a}}\right)^{2}, \\
q_{y}=\frac{1}{3 \eta}\left(h-S_{\mathrm{a}}\right)^{3} \frac{\partial p}{\partial y}+\frac{1}{2 \eta} \frac{\partial \gamma}{\partial y}\left(h-S_{\mathrm{a}}\right)^{2} .
\end{array}\right.
$$

The Laplace pressure is given as a function of the surface derivatives by Equation 7. Using the mass conservation equation, one gets:

$$
\frac{\partial h}{\partial t}+\frac{\partial q_{x}}{\partial x}+\frac{\partial q_{y}}{\partial y}+E=0
$$

In Equation 18, as the concentration locally vary, the evaporation rate varies both in time and in space. The equation governing the concentration $c$ is obtained by using the solvent 
mass conservation equation:

$$
\frac{\partial(c e)}{\partial t}=-E-\frac{\partial\left(c q_{x}\right)}{\partial x}-\frac{\partial\left(c q_{y}\right)}{\partial y}
$$

hence using Equation 18:

$$
\frac{\partial c}{\partial t}=-\left(\frac{1-c}{e}\right) E-\left(\frac{\partial c}{\partial x}\right) \frac{q_{x}}{e}-\left(\frac{\partial c}{\partial y}\right) \frac{q_{y}}{e} .
$$

The combination of Equations 18 and 20 completely describes the evolution of the film topography. The physical parameter $\gamma$ is related to the solvent concentration by the law presented later on Figure 7.

\subsection{Numerical implementation}

The leveling of the paint layer is described by high order non-linear partial differential equations. The numerical handling of these equations is therefore a delicate problem. The model Equations 15, 18 and 20 can be written in the form:

$$
\frac{\partial \psi}{\partial t}=F\left(\psi, \frac{\partial \psi}{\partial x}, \frac{\partial \psi}{\partial y}, \ldots, \frac{\partial^{n} \psi}{\partial x^{n}}, \frac{\partial^{n} \psi}{\partial y^{n}}, \ldots\right)
$$

where $F$ is a non-linear function of the spatial derivatives. The method of lines [18] is used to solve Equation 21, in combination with a pseudo-spectral method: Function $F$ is evaluated in the Fourier space and Equation 21 is integrated using an adaptative step size Runge-Kutta scheme.

\subsubsection{Evaluation of spatial gradients}

We assume that Equation 21 is submitted to periodic spatial boundary conditions. Using the Fourier transform helps us computing high-order space derivatives present in Equations 15,18 , and 20 in a simple way. However the Fourier transform of a product of functions in physical space is the convolution of the Fourier transforms of the functions. Numerically, care has to be taken when the Fourier transform of the product is calculated, since sampling implies aliasing. Let $f$ and $g$ be two functions which are sampled with a step equal to one. The Fourier series expansion of these functions are

$$
f[n]=\sum_{k=-N / 2}^{N / 2} \hat{f}[k] e^{i \frac{2 \pi}{N} k n}, \quad g[n]=\sum_{k=-N / 2}^{N / 2} \hat{g}[k] e^{i \frac{2 \pi}{N} k n} .
$$

A consequence of the function sampling is that its Fourier transform is artificially periodized. Considering Equation 22, the Fourier series expansion of the product function $f g$ is

$$
\hat{f g}[k]=\sum_{n=0}^{N} f g[n] e^{-i \frac{2 \pi}{N} k n}=\sum_{n=0}^{N} \sum_{k_{1}, k_{2}=-N / 2}^{N / 2} \hat{f}\left[k_{1}\right] \hat{g}\left[k_{2}\right] e^{\left(-i \frac{2 \pi}{N}\left(k-k_{1}-k_{2}\right) n\right)}
$$


The quantity $\sum_{n=0}^{N} e^{\left(-i \frac{2 \pi}{N}\left(k-k_{1}-k_{2}\right) n\right)}$ cancels for all values of $k, k_{1}$ and $k_{2}$, except when $k=$ $k_{1}+k_{2}+m N$, with $m \in \mathbb{Z}$. Considering the values taken by $k, k_{1}$ and $k_{2}$, we verify that

$$
\begin{aligned}
\hat{f g}[k]= & \sum_{k_{1}=-N / 2}^{N / 2} \hat{f}\left[k_{1}\right] \hat{g}\left[k-k_{1}\right]+\sum_{k_{1}=-N / 2}^{N / 2} \hat{f}\left[k_{1}\right] \hat{g}\left[k+N-k_{1}\right] \\
& +\sum_{k_{1}=-N / 2}^{N / 2} \hat{f}\left[k_{1}\right] \hat{g}\left[k-N-k_{1}\right] .
\end{aligned}
$$

The first term on the right hand side of Equation 24 corresponds to the convolution product of the Fourier transforms of $f$ and $g$. The two other terms arise from aliasing and have to be removed. To do this, a simple method is to consider $M$ frequencies instead of $\mathrm{N}$, with $N<M$, where all terms whose frequencies belong to the intervals ] $-\frac{M}{2},-\frac{N}{2}$ [ and ]$\frac{N}{2}, \frac{M}{2}$ [, are cancelled [19]. This method simply consists in oversampling the projection of our function on the basis constituted by the $N$ initial harmonics, from a spatial sampling step of size $\frac{L}{N}$ to a spatial sampling step of size $\frac{L}{M}$ :

$$
f[n]=\sum_{k=-M / 2}^{M / 2} \hat{f}[k] e^{i \frac{2 \pi}{M} k n}, \quad g[n]=\sum_{k=-M / 2}^{M / 2} \hat{g}[k] e^{i \frac{2 \pi}{M} k n} .
$$

As the frequencies between $N / 2$ and $M / 2$ are equal to zero, Equation 24 reads

$$
\begin{aligned}
\hat{f g}[k]= & \sum_{k_{1}=-N / 2}^{N / 2} \hat{f}\left[k_{1}\right] \hat{g}\left[k-k_{1}\right]+\sum_{k_{1}=-N / 2}^{N / 2} \hat{f}\left[k_{1}\right] \hat{g}\left[k+M-k_{1}\right] \\
& +\sum_{k_{1}=-N / 2}^{N / 2} \hat{f}\left[k_{1}\right] \hat{g}\left[k-M-k_{1}\right] .
\end{aligned}
$$

The most dangerous term considering aliasing is obtained for $k=-N / 2$ and $k_{1}=-N / 2$ (respectively $k=N / 2$ and $k_{1}=N / 2$ ) in the second (respectively third) sum of Equation 26. The corresponding value of $\hat{g}$ will be equal to zero if

$$
M>\frac{3 N}{2} \text {. }
$$

This inequality ensure that the quantities $k-M-k_{1}$ and $k+M-k_{1}$ fall into the intervals ] $-\frac{M}{2},-\frac{N}{2}$ [ and $] \frac{N}{2}, \frac{M}{2}$ [. The argument is easily extended to higher degree nonlinearities. Since Equation 15 involves fourth-degree monomials, full desaliasing requires $M=\frac{5 N}{2}$.

\subsubsection{Integration of the equation}

Equation 21 is integrated using a Runge-Kutta scheme. This scheme uses evaluations of the time derivative at intermediate points to achieve the integration, given by the formula:

$$
\psi_{n+1}=\psi_{n}+\Delta t \sum_{i=1}^{s} b_{i} k_{i}
$$


Table 1 General Butcher table of a Runge-Kutta scheme.



Table 2 Butcher table of the Heun scheme.

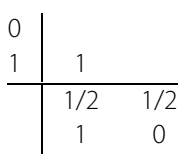

with:

$$
\left\{\begin{array}{l}
k_{1}=F\left(t_{n}, \psi_{n}\right), \\
k_{2}=F\left(t_{n}+c_{2} \Delta t, \psi_{n}+a_{21} \Delta t k_{1}\right), \\
k_{3}=F\left(t_{n}+c_{3} \Delta t, \psi_{n}+a_{31} \Delta t k_{1}+a_{32} \Delta t k_{2}\right), \\
\cdots \\
k_{N}=F\left(t_{n}+c_{s} \Delta t, \psi_{n}+a_{N 1} \Delta t k_{1}+a_{N 2} \Delta t k_{2}+\cdots+a_{N, N-1} \Delta t k_{N-1}\right) .
\end{array}\right.
$$

To specify a particular method, one simply has to set the coefficients $a_{i j}, b_{i}$ and $c_{i}$ which characterize the discretization of the equation for $i=1,2, \ldots, N$ and $j=1,2, \ldots, i$. The selected coefficients can be represented in a table called the Butcher table.

Consistency of the scheme is ensured if $\sum_{j=0}^{i} a_{i j}=c_{i}$. A Runge-Kutta scheme of order $N$ is accurate at order $N$ in $\Delta t$. It is possible to control the approximation error at each step by estimating the difference between approximations at order $N-1$ and $N$. By wisely choosing the coefficients $a_{i j}$ and $c_{i}$, intermediate points calculated in the method can be used to calculate two separate evaluations of the solution:

- A first evaluation $\psi_{n+1}=\psi_{n}+\Delta t \sum_{i=1}^{N} b_{i} k_{i}$ accurate at order $N$.

- A second evaluation $\psi_{n+1}=\psi_{n}+\Delta t \sum_{i=1}^{N-1} b^{*}{ }_{i} k_{i}$ accurate at order $N-1$, which uses an other ponderation $\left\{b^{*}{ }_{i}\right\}, i=1,2, \ldots, N$.

The difference between these two evaluations gives an estimate of the approximation error of the scheme:

$$
\epsilon=\Delta t \sum_{i=1}^{N-1}\left(b_{i}-b^{*}{ }_{i}\right) k_{i} .
$$

The corresponding Butcher table is given in Table 1.

The Heun scheme (order 2), the Bogacki-Shampine scheme [20] (order 3) and the CashKarp scheme [21] (order 5) were implemented. All these methods realize an explicit integration of Equation 21, and the schemes are conditionnaly stable. Tables 2, 3, and 4 show the Butcher tables of the schemes.

The dynamics of the paint levelling varies considerably during the painting process, and it is then of interest to use an adaptive stepsize integration scheme. A method described 
Table 3 Butcher table of the Bogacki-Shampine scheme.

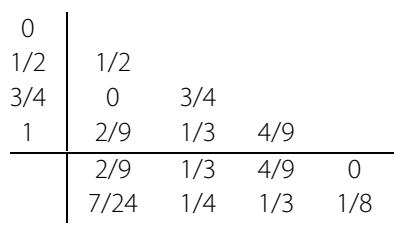

Table 4 Butcher table of the Cash-Karp scheme.

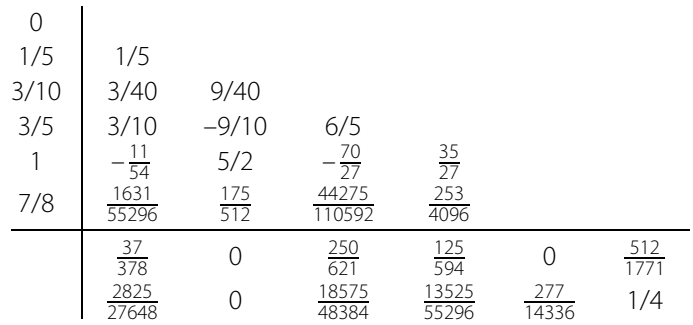

in [22] is used to adjust the time step, which uses the error estimate returned by the integration scheme.

\subsection{Validation of the numerical scheme}

Assuming that the amplitude of surface modulation is small, Equation 15 can be linearized by setting $h=h_{0}+\delta h$, expanding it in powers of $\delta h$, and keeping lowest order terms. Denoting the mean paint thickness as $e_{0}$, Equation 15 reads:

$$
\begin{aligned}
& \frac{\partial \delta h}{\partial t}(x, y, t) \\
& \quad=-\frac{\gamma}{3 \eta} e_{0}^{3}\left(\frac{\partial^{4} \delta h}{\partial x^{4}}(x, y, t)+2 \frac{\partial^{4} \delta h}{\partial x^{2} \partial y^{2}}(x, y, t)+\frac{\partial^{4} \delta h}{\partial y^{4}}(x, y, t)\right),
\end{aligned}
$$

which can be solved analytically using Fourier transforms. If Equation 31 has a solution in $L^{2}(\mathbb{R})$, in the Fourier space it fulfills:

$$
\frac{\partial \widehat{\delta h}}{\partial t}=-\frac{\gamma}{3 \eta} e_{0}^{3}\left(\xi_{x}^{4}+\xi_{x}^{2} \xi_{y}^{2}+\xi_{y}^{4}\right) \widehat{\delta h}
$$

in which $\xi=\left(\xi_{x}, \xi_{y}\right)$ is the Fourier wavevector. Consequently,

$$
\widehat{\delta h}(t)=\widehat{\delta h}(0) \exp \left(-\frac{\gamma}{3 \eta} e_{0}^{3}\left(\xi_{x}^{4}+\xi_{x}^{2} \xi_{y}^{2}+\xi_{y}^{4}\right) t\right)
$$

and

$$
\delta h(t)=\frac{1}{4 \pi^{2}} \int_{\mathbb{R}^{2}} \widehat{\delta h}(0) \exp \left(-\frac{\gamma}{3 \eta} e_{0}^{3}\left(\xi_{x}^{4}+\xi_{x}^{2} \xi_{y}^{2}+\xi_{y}^{4}\right) t\right) \exp \left(i\left(\xi_{x} x+\xi_{y} y\right)\right) \mathrm{d} \boldsymbol{\xi}
$$

Figure 3 compares the results obtained for a cataphoresis layer at $t=600$, from the initial condition displayed in the top panel, derived from the analytic solution Equation 34 (bottom-left) or obtained with the numerical scheme (bottom-right). It can be noticed that the results are very close, which validates the numerical scheme used to solve Equation 31. The parameters chosen for the comparison are given in Table 5, taken from the 

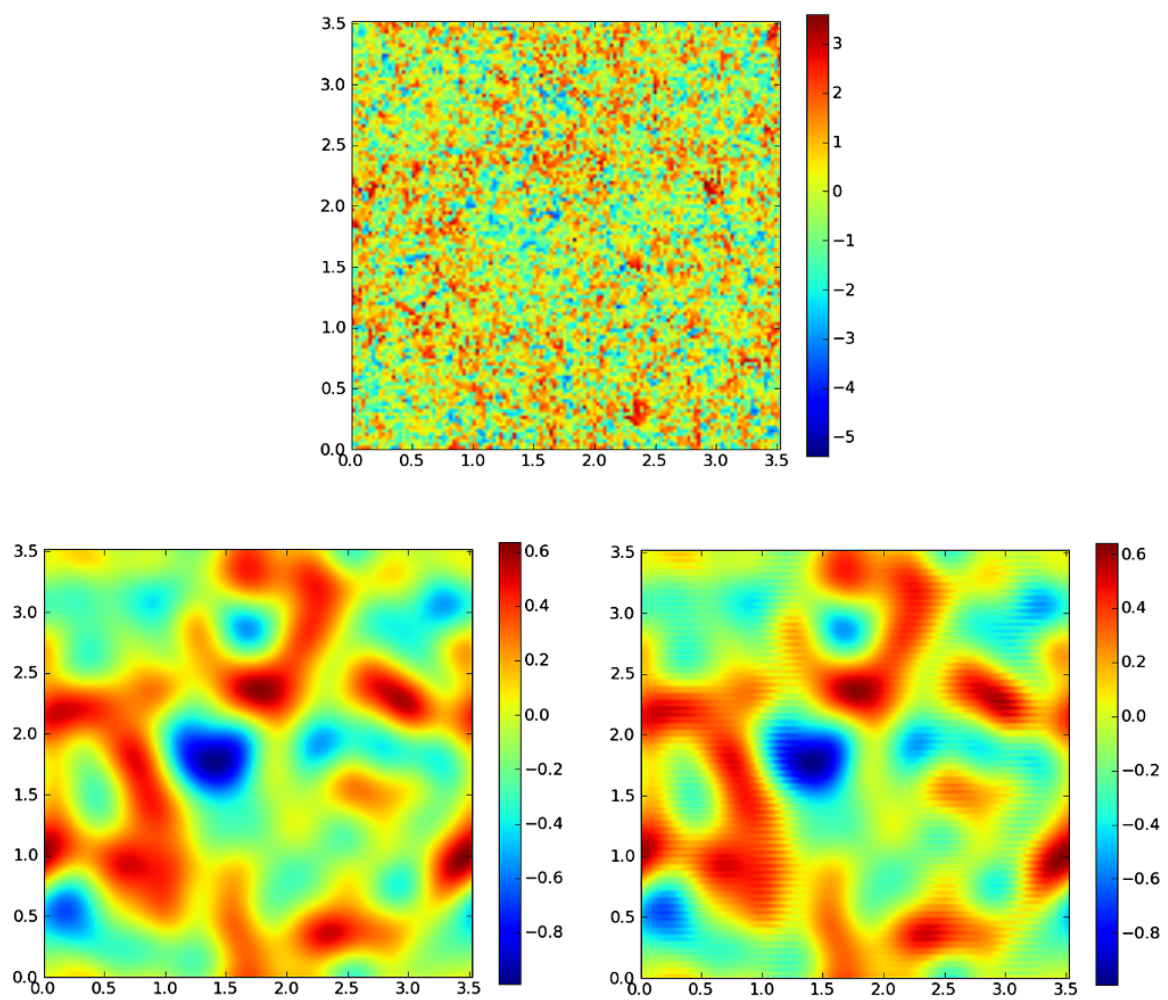

Figure 3 Validation of the numerical scheme. Top: Initial topography as measured using the Phasics ${ }^{\text {TM }}$ interferometric microscope. Bottom: Solution at $t=600$, analytical (left) and numerical (right). Each image corresponds to the same $3.52 \times 3.52 \mathrm{~mm}^{2}$ domain decomposed into $27.5 \times 27.5 \mu \mathrm{m}^{2}$ subdomains or pixels, over which the altitude is averaged (color coding).

Table 5 Physical parameters used for the numerical scheme validation.

\begin{tabular}{lccc}
\hline Parameter & Symbol & Value & Unit \\
\hline Surface tension & $\gamma$ & $3.0 \times 10^{-2}$ & $\mathrm{~N} / \mathrm{m}$ \\
Paint viscosity & $\eta$ & 1.0 & Pa.s \\
Initial thickness & $e_{0}$ & 20.0 & $\mu \mathrm{m}$ \\
\hline
\end{tabular}

relevant literature [2]. It is also assumed that the surface tension is constant and that the substrate is perfectly flat $\left(S_{\mathrm{a}}(x, y)=0\right.$ everywhere).

\section{Experimental measurements}

We now present the experiments performed with the high resolution wavefront sensor developed by Phasics ${ }^{\text {Tw }}[10]$, using a technology based on a modified Hartmann test to measure wavefront distortions: by means of $2 \mathrm{D}$ diffraction grating, a beam is replicated into four identical waves which are propagated along slightly different directions. The direction differences create interference patterns and the interference fringes are used to reconstruct the measured surface topography. We use it to map painted steel sheets at regular time intervals during the whole painting process, for a sealer or a lacquer layer. The experimental procedure is as follows:

- Paint is deposited over a sample of metal sheet (polished or already covered with an electrophoresis layer) in a painting cabin using a paint gun. 
- The sheet is then placed on a baking plate. During the first few minutes, complete samplings of the surface are performed at regular time intervals (typically $2.5 \mathrm{~Hz}$ ), in order to record the evolution of the painted layer topography at the beginning of the flash time in detail.

- After two minutes the sampling rate is decreased to $0.1 \mathrm{~Hz}$, for the flow dynamic next slows down considerably.

- The baking cycle starts after 10 minutes, with the sampling frequency reincreased to $1.25 \mathrm{~Hz}$.

- Chemical bonds begin to form within the paint 5 minutes after the beginning of the baking. Cross-linking then stops the evolution so that the sampling frequency can be decreased to $0.1 \mathrm{~Hz}$.

The wavefront sensor collects information over a surface of $18 \times 18 \mathrm{~mm}^{2}$. The topography is analyzed as a $128 \times 128$ square image. Each pixel represents the mean altitude over a $60 \times 60 \mu \mathrm{m}^{2}$ surface. The precision of vertical measurements is up to $10^{-2} \mu \mathrm{m}$.

During the flash time, the outside temperature is $25^{\circ} \mathrm{C}$. The baking cycle is divided into two stages: a linear temperature rise during $300 \mathrm{sec}$ until reaching $150^{\circ} \mathrm{C}$, followed by a 15 minutes plateau at this temperature.

\subsection{Surface evolution}

The following figures show the evolution of the topography of a lacquer layer during the whole painting process. The lacquer is deposited on a smooth substrate. Altitudes are given in $\mu \mathrm{m}$. On each surface, during measurements, the minimum is arbitrarily set to zero since only relative but not absolute altitudes can be obtained from the device.

Figure 4 displays the beginning of the flash time. A rapid leveling of the paint is observed, due to the combined effects of the rapid evaporation of the light sealer solvent and the flow caused by surface tension. The phenomenon is specially important at the beginning of the flash time when the viscosity of the paint is still relatively low. At the end of the flash time, the leveling slows down until the topography of the layer stops evolving.

Figure 5 shows the evolution of the lacquer layer during the baking. The same altitude scale has been kept, which allows a comparison with the previous sequence. A second stage of leveling and evaporation takes place during the baking of the lacquer. Temperature increase promotes the evaporation of heavier solvents contained in paint and the subsequent cross-linking of the molecules.

\subsection{Evolution of the roughness}

Roughness evolution during the painting process helps us quantifying the paint leveling capability. Since the physical effects involved develop at different scales, it is of interest to play with tools able to separate the different roughness scales. The surface is sampled with a $60 \mu \mathrm{m}$ horizontal step, yielding a $128 \times 128$ image $S=S\left[n_{1}, n_{2}\right]$. An algorithm based on the wavelet packet transform [23] and the reconstruction formula is used, that allows a decomposition of the roughness into a sum of contributions [24-26].

A wavelet $\psi(t)$ is a function in $L^{2}(\mathbb{R})$ which has zero average, such that $\|\psi\|=1$ using the $L^{2}$ norm, and centered around $t=0$ [23]. We obtain a wavelet family by dilating this function with a scale parameter $s>0$ and translating it by $u \in \mathbb{R}$ :

$$
\psi_{u, s}(t)=\frac{1}{\sqrt{s}} \psi\left(\frac{t-u}{s}\right) .
$$



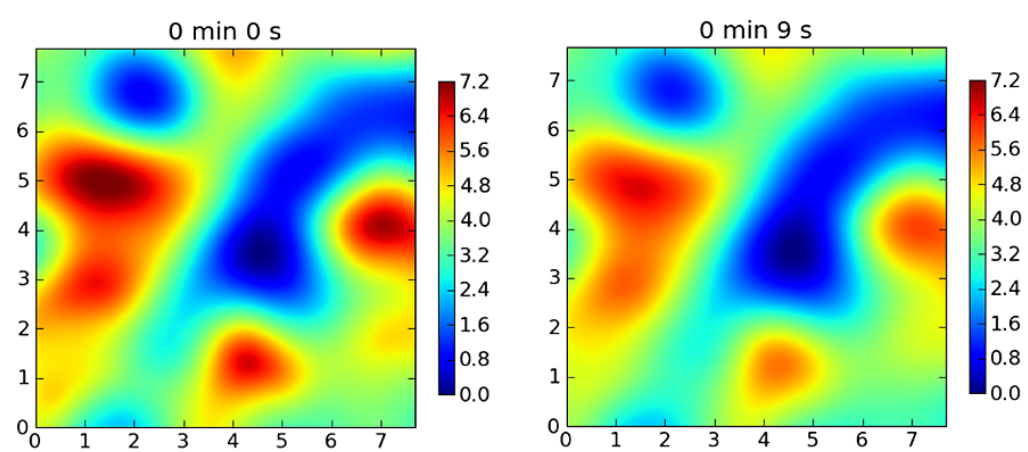

$0 \min 19 \mathrm{~s}$
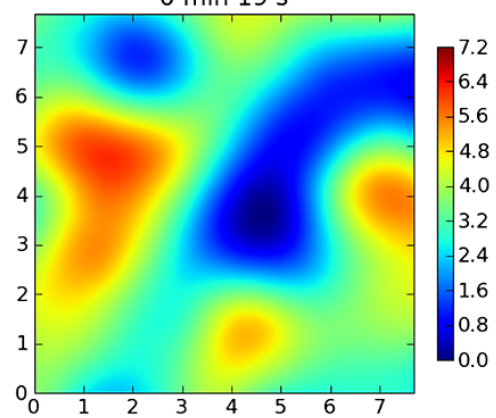

$1 \min 41 \mathrm{~s}$
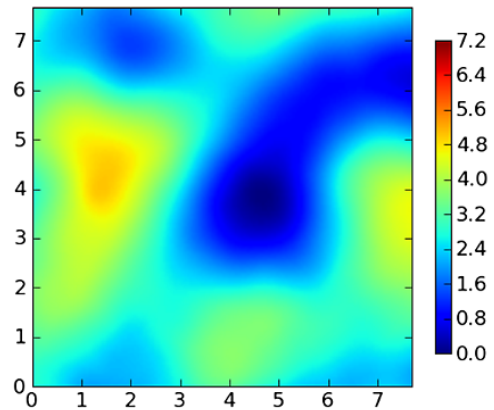

$0 \min 45 \mathrm{~s}$

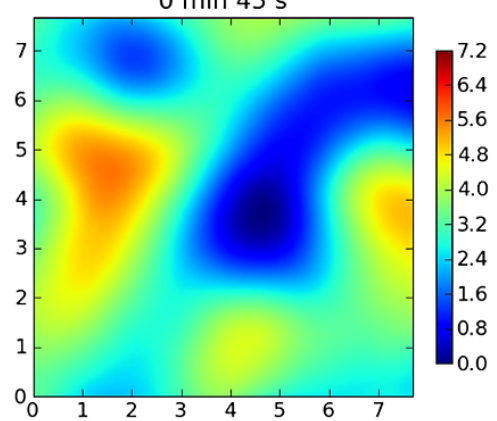

$11 \min 12 \mathrm{~s}$

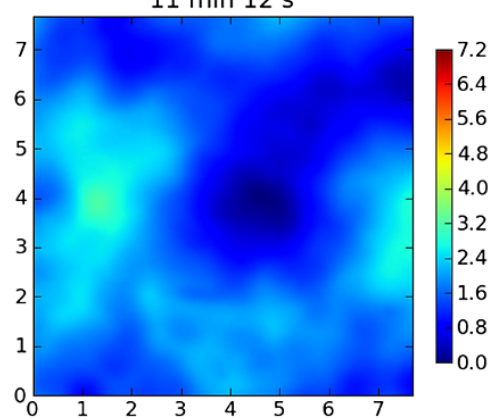

Figure 4 Evolution of the topography of the lacquer film during the flash time. The topography of a $7.68 \times 7.68 \mathrm{~mm}^{2}$ surface is represented in a $128 \times 128$ square image. Color coding at each pixel gives the mean altitude over a $60 \times 60 \mu \mathrm{m}^{2}$ surface.

Let $f$ be a function in $L^{2}(\mathbb{R})$. The continuous wavelet transform of $f$ is the function:

$$
W f(u, s)=\int_{-\infty}^{\infty} f(t) \frac{1}{\sqrt{s}} \psi\left(\frac{t-u}{s}\right) \mathrm{d} t
$$

The original signal can be reconstructed from its wavelet coefficients:

$$
f(t)=\frac{1}{C_{\psi}} \int_{0}^{\infty} \int_{-\infty}^{\infty} W f(u, s) \frac{1}{\sqrt{s}} \psi\left(\frac{t-u}{s}\right) \mathrm{d} u \frac{\mathrm{d} s}{s^{2}},
$$

where

$$
C_{\psi}=\int_{0}^{\infty} \frac{|\hat{\psi}(\omega)|^{2}}{\omega} \mathrm{d} \omega<+\infty
$$






A wavelet is a function which is well localized both in physical and Fourier spaces. The projection of the studied surface on a family of wavelets allows us to identify the scale hierarchy present in the pattern. The scale-by-scale reconstruction of the surface can next isolate the different contributions to the roughness of the given topography. The continuous wavelet transform was adapted to the discrete case, yielding the orthonormal wavelet and wavelet packet transforms [23]. The roughness characterization algorithm therefore decomposes the studied surface $S\left[n_{1}, n_{2}\right]$ onto a family of wavelet packets, and next reconstructs it by keeping only wavelet coefficients corresponding to successive scale:

$$
S\left[n_{1}, n_{2}\right]=\sum_{j=0}^{J} S_{j}\left[n_{1}, n_{2}\right],
$$

$J$ denoting the number of scales and $S_{j}$ the reconstructed surface at the scale $j$. For each reconstructed surface, it is possible to define a parameter $M q$ characterizing the mean deviation from the average surface at scale $j$. The resulting curve $M q[j]$ then accounts for the frequency content of the studied roughness:

$$
M q[j]=\frac{1}{N^{2}} \sqrt{\sum_{n_{1}=1}^{N} \sum_{n_{2}=1}^{N}\left(S_{j}\left[n_{1}, n_{2}\right]-E\left[S_{j}\right]\right)^{2}} .
$$

Figures 6 show how $M q$ changes from one to another step of the painting process. It is interesting to note that the small scales are not completely attenuated during the painting process, due to resurgence of the underlying substrate residual roughness. On the other hand, the baking has little impact on the paint leveling for the lacquer.

The scale-by-scale study of the surface roughness provides valuable information on the dynamics of the leveling. The curves in Figure 6 show little leveling during the baking, the difference being mainly due to evaporation since the two curves are quite similar. In the next section we therefore focus only on the simulation of the surface dynamics during flash time, when both flow and evaporation are involved. 


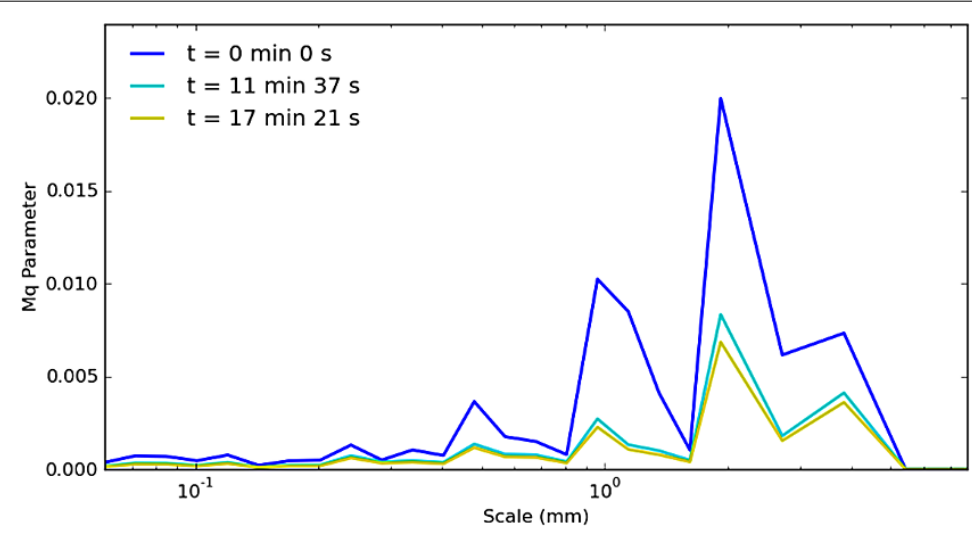

Figure 6 Evolution of $M q$ as a function of the scale during the whole painting process.

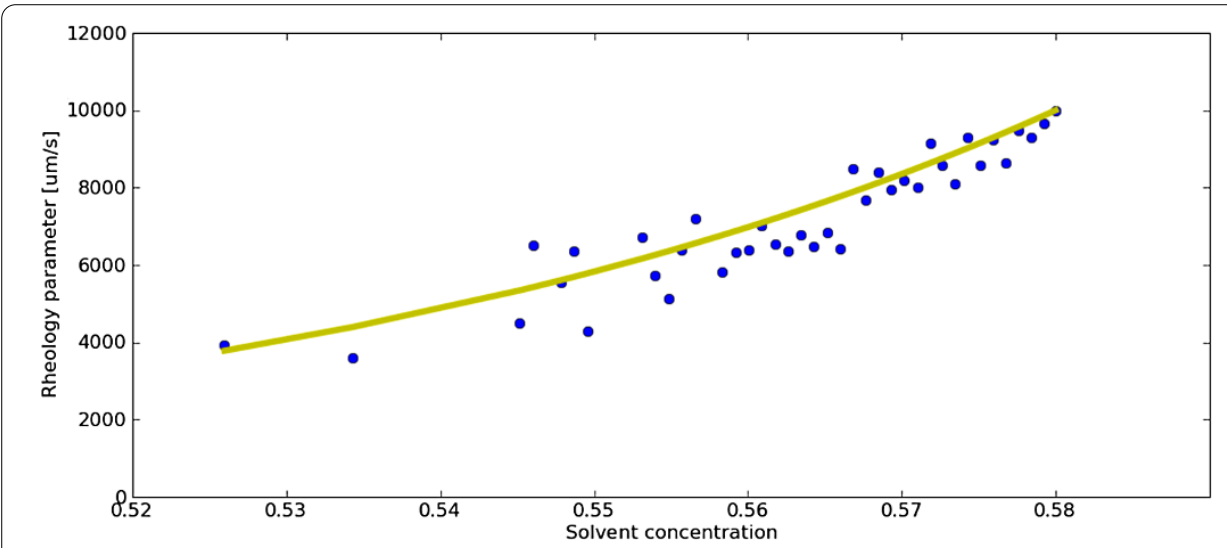

Figure 7 Variation of $\frac{\gamma}{3 \eta}$ as a function of the solvent concentration.

Table 6 Simulation parameters.

\begin{tabular}{lcccc}
\hline Parameter & Symbol & Value & Value in [2] & Unit \\
\hline Resin surface tension & $\gamma_{r}$ & - & $3.0 \times 10^{-2}$ & $\mathrm{~N} / \mathrm{m}$ \\
Solvent surface tension & $\gamma_{s}$ & - & $2.5 \times 10^{-2}$ & $\mathrm{~N} / \mathrm{m}$ \\
Initial paint viscosity & $\eta_{0}$ & - & $0.55-1.59$ & Pa.s \\
Initial solvent concentration & $c_{0}$ & 0.58 & 0.5 & - \\
Viscosity exponent & $a$ & 18 & $15[5]$ & - \\
Evaporation parameter & $\lambda$ & $4.0 \times 10^{-9}$ & $2.0 \times 10^{-9}$ & $\mathrm{~m} / \mathrm{s}$ \\
Initial rheological parameter & $\gamma_{0} / 3 \eta_{0}$ & $1.0 \times 10^{4}$ & $1.0 \times 10^{4}$ & $\mu \mathrm{m} / \mathrm{s}$ \\
\hline
\end{tabular}

\section{Direct simulation}

We now compare simulation results to experimental data obtained from the wavefront sensor. In [11], the value of the rheological parameter $\gamma / 3 \eta$ was determined as a function of the solvent concentration $c$ from mass fluxes computed by solving an inverse problem relying on the Newtonian model. The surface tension is assumed to be related to the solvent concentration via:

$$
\gamma=\gamma_{r}+c\left(\gamma_{s}-\gamma_{r}\right)=\gamma_{r}+c \Delta \gamma
$$




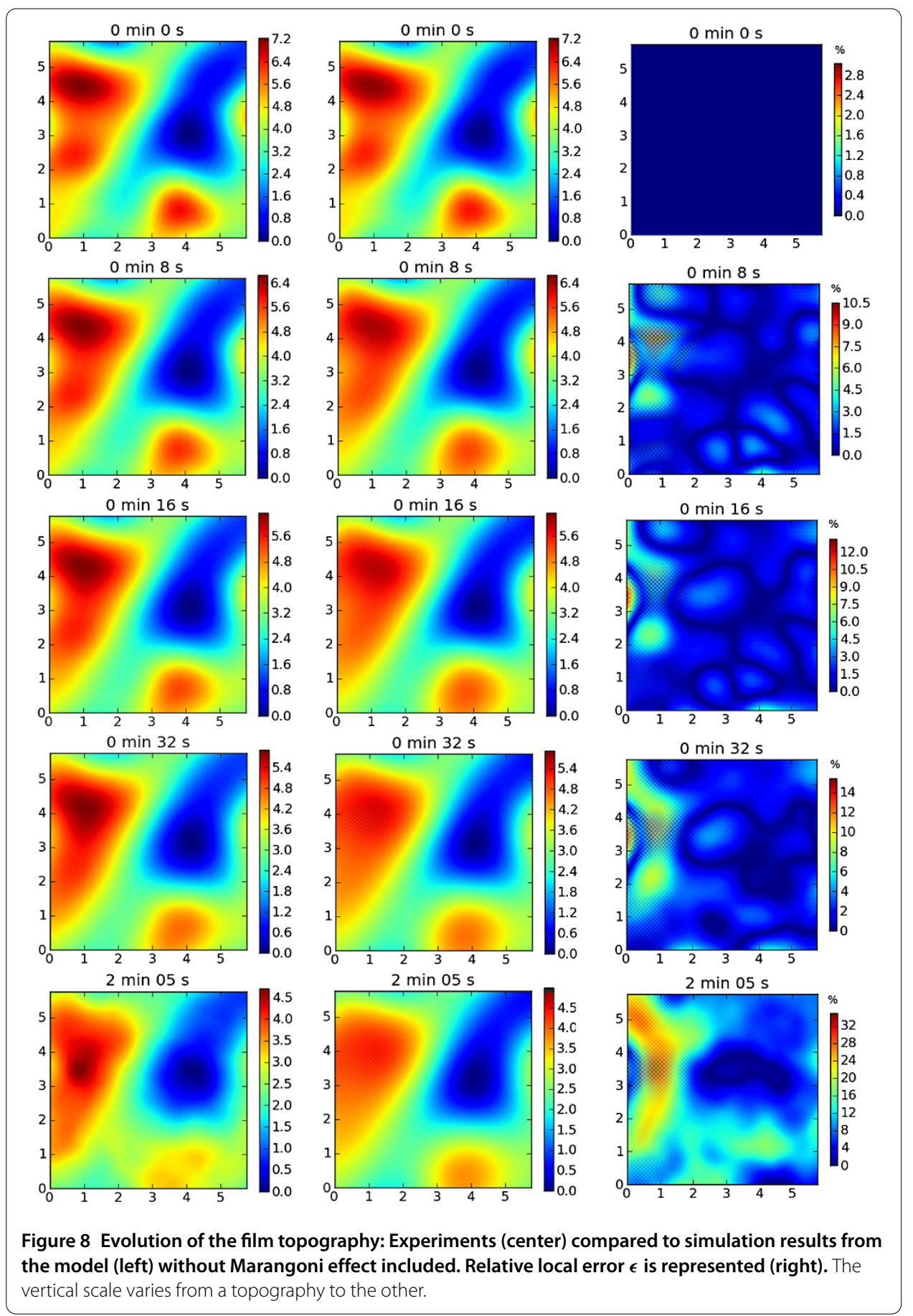

where $\gamma_{r}\left(\gamma_{s}\right)$ denotes the resin (solvent) surface tension. On the other hand, viscosity depends upon the solvent concentration according to:

$$
\eta(c)=\eta_{0} e^{-a c} .
$$

Using these relations to fit the experimental data during the flash time (Figure 7), we find:

$$
\frac{\gamma}{3 \eta}(c)=\frac{\gamma_{0}}{3 \eta_{0}} e^{a\left(c-c_{0}\right)},
$$




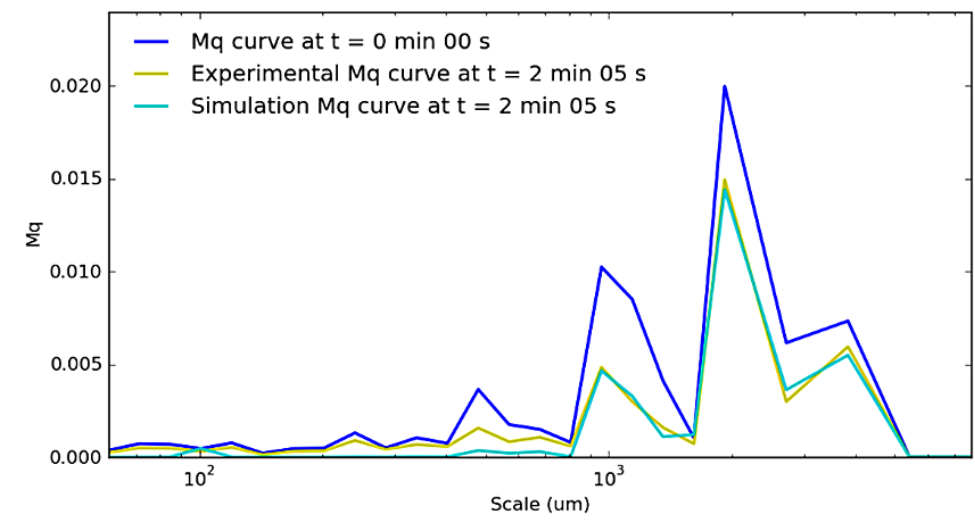

Figure 9 Evolution of the $M q$ curves: Experiments are compared to simulation results.

with $\frac{\gamma_{0}}{3 \eta_{0}}=0.1 \mathrm{~m} / \mathrm{s}, c_{0}=0.58$ and $a=18.0$. Table 6 shows that the rheological parameters close to those reported in $[2,5]$.

Using the experimental data obtained with the wavefront analyzer and the evaporation law deduced from these measurements, simulations were performed with the two models described in Section 2. These simulations start from the first reconstructed topography and aim at reproducing the entire evolution of the film during the flash time. Parameters used are given in Table 6 obtained as explained above. We consider that the substrate is completely smooth. The numerical resolution code was described at the end of Section 2. The simulations are performed using a $3.40 \mathrm{GHz}$ Intel(R) Xeon(TM) processor, and last about five hours.

The local relative error defined as

$$
\epsilon(x, y)=\frac{\left|h^{s}(x, y)-h^{e}(x, y)\right|}{\max _{(x, y)} h^{e}},
$$

where $h^{s}$ denotes the simulated topography and $h^{e}$ the experimental one is displayed in Figure 8 (right). The main error is caused by side effects, as the surface is artificially periodized. The local simulation error remains small, and the model seems to be able to reproduce the patterns evolution, at least at the millimeter scale. It remains interesting to compare the $M q$ curves of the simulated and experimental topographies. The curves of Figure 9 show that the simulation model is able to reproduce the leveling dynamics for the millimetric scales. It was supposed that the substrate of the paint layer was completely smooth. Nevertheless, the substrate exhibits some roughness at the smallest scales, and evaporation causes the resurgence of the underlying substrate roughness, which explains the differences between the simulation and the experimental $M q$ curves. For the last represented topography, the resurgence of the underlying topography also introduces an error.

Figure 10 compares simulations realized with the model including Marangoni effect (right) and not (left), showing that the model developed by Orchard [1] seems sufficiently accurate to explain the physics of the leveling of the coating. In fact, the geometric situation considered here is somewhat different from those studied by Weidner et al. [5] or Gaskell et al. [9]. For automotive paints, fluctuations in the thickness of the coating are indeed relatively small $(<7 \mu \mathrm{m})$ compared to the thickness itself $(\cong 70 \mu \mathrm{m})$, so that the 



Figure 10 Comparison between simulation results from the model with (right) or without (left) Marangoni effect included.

rate of evaporation and consequently the solvent concentration remain rather uniform in the paint layer.

\section{Conclusion}

Painting of steel sheets is a complex phenomenon that depends on many physical processes. With the wavefront sensor developed by Phasics ${ }^{\mathrm{it}}$, it was possible to perform experiments allowing an accurate monitoring of the topography of a film during its deposition. The fast response time of the wavefront sensor allowed us to access the rheological 
parameters of the paint in an original way by solving an inverse problem. The obtained parameters were used to perform a complete simulation of the film evolution during the painting process, which demonstrated that the Newtonian model was able to reproduce the leveling of the paint layer accurately and that Marangoni effect could be neglected at the beginning of the flash time, when significant flow occurs. At the end of the flash time, the flow rates decreases and it is clear that the film then exhibits a more complex rheology due to the solvent evaporation, but the leveling dynamic is then considerably attenuated, and the influence on the surface topography is negligible. The conducted simulations are however complex and require the development of advanced numerical tools, like those presented in this paper.

Competing interests

Our research was supported by ArcelorMittal Global R\&D, F-57283 Maizières-lès-Metz Cedex, France.

Author's contribution

$\mathrm{BF}, \mathrm{DJ}$ and GF designed and implemented the numerical scheme which is presented in this paper. The experiments were realized by BF, GF and J-JP. The physical models and the resolution of the inverse problem were tackled by BF, DJ, AL and PM. All authors participated in writing the manuscript.

\section{Author details}

'Centre de Morphologie Mathématique, École des Mines ParisTech, 77300, Fontainebleau, France. ${ }^{2}$ UMR Navier, 2 allée Kepler, 77420, Champs-sur-Marne, France. ${ }^{3}$ ArcelorMittal Global R\&D, 57283, Maizières-lès-Metz Cedex, France. ${ }^{4}$ LadHyX, École Polytechnique, 91128, Palaiseau, France.

\section{Received: 4 May 2011 Accepted: 3 January 2012 Published: 3 January 2012}

\section{References}

1. Orchard SE: On surface levelling in viscous liquids and gels. App/ Sci Res 1961, 11:451-464.

2. Overdiep WS: The levelling of paints. Prog Org Coat 1986, 14:159-175.

3. Wilson SK: The levelling of paint films. IMA J App/ Math 1993, 50:149-166.

4. Howison SD, Moriarty JA, Ockendon JR, Terrill EL: A mathematical model for drying paint layers. J Eng Math 1997, 32:377-394.

5. Weidner DE, Schwartz LW, Eley RR: Role of surface tension gradients in correcting coating defects in corners J Colloid Interface Sci 1996, 179:66-75.

6. Eres MH, Weidner DE, Schwartz LW: Three-dimensional direct numerical simulation of surface-tension-gradient effects on the leveling of an evaporating multicomponent fluid. Langmuir 1999, 15:1859-1871.

7. Schwartz LW, Roy RV, Eley R, Petrash S: Dewetting patterns in a drying liquid film. J Colloid Interface Sci 2001, 234:363-374

8. Gaskell PH, Jimack PK, Sellier M, Thompson HM, Wilson MCT: Gravity-driven flow of continuous thin liquid films on non-porous substrates with topography. J Fluid Mech 2004, 509:253-280.

9. Gaskell PH, Jimack PK, Sellier M, Thompson HM: Flow of evaporating gravity-driven thin liquid films over topography. Phys Fluids 2006, 18:031601

10. Phasics: [http://www.phasicscorp.com/]

11. Figliuzzi B, Jeulin D, Lemaitre A, Manneville P, Fricout G, Piezanowski JJ: Rheology of thin films from flow observations. Exp. Fluids, submitted.

12. Benney DJ: Long waves on liquid films. J Math Phys 1966, 45:150-155.

13. Shkadov VY: Wave flow regimes of a thin layer of viscous fluid subject to gravity. IzV AN SSSR Mekhanika Zhidkosti i Gaza 1967, 2(1):43-51.

14. Shkadov VY: Solitary waves in a layer of viscous liquid. IzV AN SSSR Mekhanika Zhidkosti i Gaza 1977, 1:63-66.

15. Ruyer-Quil C, Manneville P: Improved modeling of flows down inclined planes. Eur Phys J B 2000, 15:357-369.

16. Ruyer-Quil C, Manneville P: Modeling film flows down inclined planes. Eur Phys J B 1998, 6:277-292.

17. Oron A, Davis SH, Bankoff SG: Long scale evolution of thin liquid films. Rev Mod Phys 1997, 69(3):931-980.

18. Fletcher CAJ: Computational Techniques for Fluid Dynamics. Berlin: Springer; 1991.

19. Manneville P: Instabilities, Chaos and Turbulence. London: Imperial College Press; 2010.

20. Bogacki P, Shampine L: A 3(2) pair of Runge-Kutta formulas. App/ Math Lett 1989, 2:321-325.

21. Cash JR, Karp AH: A variable order Runge-Kutta method for initial value problems with rapidly varying right-hand sides. ACM Trans Math Softw 1990, 16:201-222.

22. Press WH, Teukolsky SA, Vetterling WT, Flannery BP: Numerical Recipes: The Art of Scientific Computing. 3rd edition. Cambridge: Cambridge University Press; 2007.

23. Mallat S: A Wavelet Tour of Signal Processing. San Diego: Academic Press; 2008.

24. Lee SH, Zahouani H, Caterini R, Mathia TG: Morphological characterization of engineered surfaces by wavelet transform. Int J Mach Tools Manuf 1998, 38:581-589.

25. Chen Q, Yang S, Li Z: Surface roughness evaluation by using wavelets analysis. Precis Eng 1999, 23:209-212. 
26. Liu JJ, Kim D, Han C: Use of wavelet packet transform in characterization of surface quality. Ind Eng Chem Res 2007, 46:5152-5158.

doi:10.1186/2190-5983-2-1

Cite this article as: Figliuzzi et al.: Numerical simulation of thin paint film flow. Journal of Mathematics in Industry 2012 2:1 